\title{
Interleukin-4 and CpG oligonucleotide therapy suppresses the outgrowth of tumors by activating tumor-specific Th1-type immune responses
}

\author{
ATSUSHI KAJIWARA* ${ }^{*}$, HIROYOSHI DOI ${ }^{*}$, JUNICHI EGUCHI, \\ SHIGEAKI ISHII, AYAKO HIRAIDE-SASAGAWA, MASASHI SAKAKI, RISA OMORI, \\ KAZUMASA HIROISHI and MICHIO IMAWARI
}

Division of Gastroenterology, Department of Medicine, Showa University School of Medicine, Tokyo 142-8666, Japan

Received November 14, 2011; Accepted January 9, 2012

DOI: 10.3892/or.2012.1723

\begin{abstract}
Because IL-4 and CpG oligodeoxynucleotides (CpG-ODNs) are immune stimulants, we evaluated the antitumor effects of IL-4 gene therapy and CpG-ODN treatment in a poorly immunogenic murine cancer model. We used a murine colorectal cancer MC38 cell line overexpressing the IL-4 gene (MC38-IL4). Incubation with MC38-IL4 and CpG-ODN enhanced bone marrow-derived dendritic cell (DC) maturation in vitro. In addition, interferon (IFN)- $\gamma$ production was significantly increased in naïve splenocytes after they were coincubated with MC38-IL4 and CpG-ODN. When mice bearing MC38 wild-type tumors were inoculated subcutaneously with MC38-IL4 cells and CpG-ODN, the outgrowth of established parental tumors was significantly suppressed compared to those in the MC38-IL4-treated group (IL-4 vs. IL-4 + CpG-ODN, $\mathrm{P}=0.015$ ). A marked infiltration of $\mathrm{CD}^{+}$cells in the established parental tumors of mice treated with MC38-IL4 and CpG-ODN was confirmed by immunohistochemical analyses (MC38-IL4, 2.8 \pm 1.9 cells/field vs. MC38-IL4 + CpG-ODN, 20.7 \pm 15.3 cells/field, $\mathrm{P}=0.027$ ). Significant tumor-specific cytolysis was detected when splenocytes of MC38-IL4 + CpG-ODN-treated mice were stimulated by $\gamma$-irradiated MC38-IL4 cells and CpG-ODN twice weekly in vitro and used as effector cells in a chromium-release assay (32.2 $\pm 3.5 \%$ for MC38 cells vs. $3.2 \pm 1.1 \%$ for YAC-1 cells; at an effector to target ratio of 40). These results suggest that IL-4 and $\mathrm{CpG}-\mathrm{ODN}$ treatment promotes potent Th1-type antitumor immune responses. Therefore, the combination of IL-4 gene
\end{abstract}

Correspondence to: Dr Kazumasa Hiroishi, Division of Gastroenterology, Department of Medicine, Showa University School of Medicine, 1-5-8 Hatanodai, Shinagawa-ku, Tokyo 142-8666, Japan E-mail: hiroishi@med.showa-u.ac.jp

${ }^{*}$ Contributed equally

Key words: interleukin-4, CpG, Th1, cytotoxic T lymphocyte, colorectal cancer therapy and CpG-ODN treatment for cancer should be evaluated in clinical trials.

\section{Introduction}

Several original treatments of cancer have been developed in the last decade. Many anticancer drugs, including moleculetargeted drugs, have been applied clinically. Although some of these drugs have dramatically changed the prognoses of a number of diseases, several harmful side effects were observed. Because cancer immunotherapy is thought to induce few adverse events compared to other therapies, such as chemotherapy and radiation, the establishment of novel treatments that elicit potent immune responses against cancer has been anticipated. Indeed, many clinical trials of such treatments have been conducted. However, most of them have shown only minimal effects, and immunotherapy has not yet been applied as a conventional clinical treatment for cancer. Moreover, patients with advanced cancer exhibit impaired immune responses. Therefore, new strategies are needed to induce more potent antitumor immunities.

We have previously reported the antitumor effects and mechanisms of cell-based immunotherapies that were combined with treatments with cytokines, such as interferon (IFN)- $\alpha$ (1-3), IL-4 (4) and IL-12 (1). The administration of each cytokine gene-transduced tumor cells appears to induce potent cellular immune responses. However, the antitumor immune response mechanisms that were induced by these cytokine therapies differ from each other. In particular, we reported that IL-4 treatment resulted in unique and interesting biological effects.

IL-4, which is a type-2 response inducer $(5,6)$, plays a major role in both $\mathrm{B}$-cell and T-cell development in the immune system (7). In addition, it causes a class switch of B cells, upregulates MHC class II and adhesion molecules, and prevents the apoptosis of T cells (8). With respect to the antitumor effects, IL-4 has a direct inhibitory effect on tumor cell growth in vivo and in vitro $(9,10)$ and an antiangiogenic effect (11). It has been reported that IL-4 activates endothelia in the tumor microenvironment, which results in an increased infiltration of immune cells (12). Other reports have suggested 
that eosinophils and neutrophils are responsible for the antitumor effects induced by IL-4 (13-15). In addition, a recent study demonstrated that local IL-4 delivery at the site of vaccination activates local dendritic cells (DCs), which play a critical role in the initiation, promotion, and regulation of host immune responses and promote type-1 T-helper (Th1) cell responses (16). In that investigation, IL-4 appeared to support DC maturation and enhance IL-12p70 secretion from DCs. Because these findings support the therapeutic effects of IL-4 on tumors, IL-4 has been applied in the clinical treatment of tumors $(17,18)$.

The potent immunomodulator $\mathrm{CpG}$ is present at the expected frequency of about 1 in 16 bases in bacterial DNA, but it is hardly observed in vertebrate DNA. It has been reported that DNA vaccines and synthetic oligodeoxynucleotides (ODN) containing an unmethylated $\mathrm{CpG}$ motif promote Th1-type immune responses. CpG stimulates DCs through the toll-like receptor 9 and enhances DC maturation, which may improve therapeutic effects on established tumors. Recently, we reported the synergistic effects of the combination of CpG-ODN and IFN- $\alpha$ on DC maturation (19). Furthermore, DC-based therapy with CpG-ODN and IFN- $\alpha$ exhibited potent antitumor effects by inducing tumor-specific cytotoxic T-lymphocyte (CTL) responses (3).

Although we have previously demonstrated that IL-4 promotes a Th1-type antitumor immune response, evidence of the induction of a more potent immune response that diminishes established tumors in vivo is required before justification of a clinical trial. In the present study, we anticipated that the combination of IL-4 gene therapy and CpG-ODN would have additive effects on tumors. Thus, we investigated the antitumor effects of the combination therapy and the underlying mechanisms of these effects in order to determine whether the combined therapy would be appropriated in a clinical trial.

\section{Materials and methods}

Mice. Female C57BL/6 (B6) mice that were 6-8 weeks old were purchased from Sankyo Lab Service (Tokyo, Japan) for use in experiments when they were 8-12 weeks old. Mice were maintained in an animal care facility at Showa University. This study was approved by the Ethics Committee for Animal Experiments of Showa University (permission \#2011-1111).

Cell lines, culture medium and reagents. The MC38 murine colorectal adenocarcinoma cell line (B6 mouse origin) and the YAC-1 lymphoma cell line were maintained in RPMI-1640 medium that was supplemented with $10 \%$ heat-inactivated fetal calf serum, $2 \mathrm{mM}$ L-glutamine, $100 \mathrm{IU} / \mathrm{ml}$ penicillin, and $100 \mu \mathrm{g} / \mathrm{ml}$ streptomycin (complete medium; CM) in a humidified incubator with $5 \% \mathrm{CO}_{2}$ in air at $37^{\circ} \mathrm{C}$. All cell culture reagents were purchased from Life Technologies Corp. (Gaithersburg,MD).CpG-ODN-1826 and non-CpG-ODN-1911 (used as control ODN) were synthesized by Sigma-Aldrich Japan (Tokyo, Japan). We confirmed that non-CpG-ODN-1911 did not affect DC maturation or have antitumor activity (19).

Genetically modified tumor cell lines. The MC38 tumor cells that were transduced using retroviral vectors according to standard protocols, as previously described (20), were selected for antibiotic resistance in $\mathrm{CM}$ containing $0.5 \mathrm{mg} / \mathrm{ml} \mathrm{G} 418$ (Sigma-Aldrich Corp., St. Louis, MO). We have established a IL-4-overexpressing MC38 cell line (MC38-IL4), which produces a large amount of IL-4 $\left(359.9 \pm 108.2 \mathrm{ng} / 10^{6} \mathrm{cells} / 48 \mathrm{~h}\right)$ (4). A neomycin-resistant gene-transduced MC38 (MC38-Neo) cell line was used as a control (21). The growth rate of the MC38-IL4 cells did not differ from those of MC38-wild-type (WT) or MC38-Neo cells in vitro.

Phenotypic changes of DCs after coincubation with IL-4overexpressing tumor cells and $C p G-O D N$. In order to observe the immunogenic effects of IL-4 and CpG-ODN on DC maturation, flow cytometry was performed using FACSCalibur (Nippon Becton-Dickinson Co., Ltd., Tokyo, Japan). Bone marrow-derived DCs were generated, as previously reported (2). We incubated the DCs $\left(5 \times 10^{6}-6 \times 10^{6}\right.$ cells/flask $)$ with

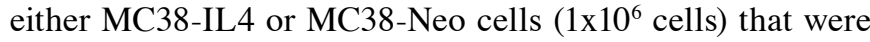
100 Gy-irradiated in combination with $6 \mu \mathrm{g} / \mathrm{ml}$ of either CpG-ODN-1826 or non-CpG-ODN-1911 in vitro. After a 24-h incubation, cells were harvested and stained with fluorescein isothiocyanate (FITC)-conjugated monoclonal antibodies. The monoclonal antibodies used in this assay were FITCconjugated anti-H-2Kb, I-Ab, CD80, and CD86 antibodies (obtained from Nippon Becton-Dickinson). The results were shown as ratios of the mean fluorescent intensity (MFI) of the DCs that were incubated with each monoclonal antibody to the MFI of DCs incubated with the FITC-conjugated control IgG antibody.

IFN- $\gamma$ and IL-10 production in naïve splenocytes coinoculated with IL-4-overexpressing tumor cells and $C p G-O D N$ in vitro. In order to assess the effects of IL-4 and CpG-ODN on immune balance, we performed an enzyme-linked immunosorbent assay (ELISA) of the representative Th1-type cytokine, IFN- $\gamma$ and the representative Th2-type cytokine, IL-10. Splenocytes $\left(1 \times 10^{6}\right.$ cells $\left./ \mathrm{ml}\right)$ from naive mice were stimulated with either MC38-IL4 or MC38-Neo cells $\left(1 \times 10^{5}\right.$ cells $\left./ \mathrm{ml}\right)$ either alone or in combination with $6 \mu \mathrm{g} / \mathrm{ml}$ of CpG-ODN-1826 or non-CpGODN-1911 in vitro. After a 24-h incubation, we measured the concentrations of IFN- $\gamma$ and IL-10 in the supernatant of each culture with ELISA using a commercially available kit, according to the manufacturer's instructions (mouse IFN- $\gamma$ ELISA and mouse IL-10 ELISA, Thermo Fisher Scientific, Inc., Rockford, IL). This experiment was repeated twice.

Combination therapy of IL-4-overexpressing tumors with $C p G-O D N$ in therapeutic models. In order to evaluate the in vivo therapeutic effects of the combination of IL-4overexpressing tumor cells and $\mathrm{CpG-ODN}$ on established tumors, we measured the size of established MC38-WT tumors in mice before and after treatment, as previously described (21). In brief, B6 mice were first injected s.c. with $3 \times 10^{5}$ MC38-WT cells in the right flank. Seven, 9, and 11 days after the WT inoculation, 3×105 MC38-Neo or MC38-IL4 cells were inoculated s.c. with $30 \mu \mathrm{g} /$ mouse of either CpG-ODN1826 or non-CpG-ODN-1911 around the established parental tumors, which had reached $4-20 \mathrm{~mm}^{2}$ in size. Otherwise, tumor-bearing B6 mice $\left(5 \times 10^{5}\right.$ MC38-WT cells/mouse) were inoculated $5 \times 10^{5} \mathrm{MC} 38-\mathrm{Neo}$ or MC38-IL4 cells were inoculated s.c. with either CpG-ODN-1826 or ODN-1911 in the same 

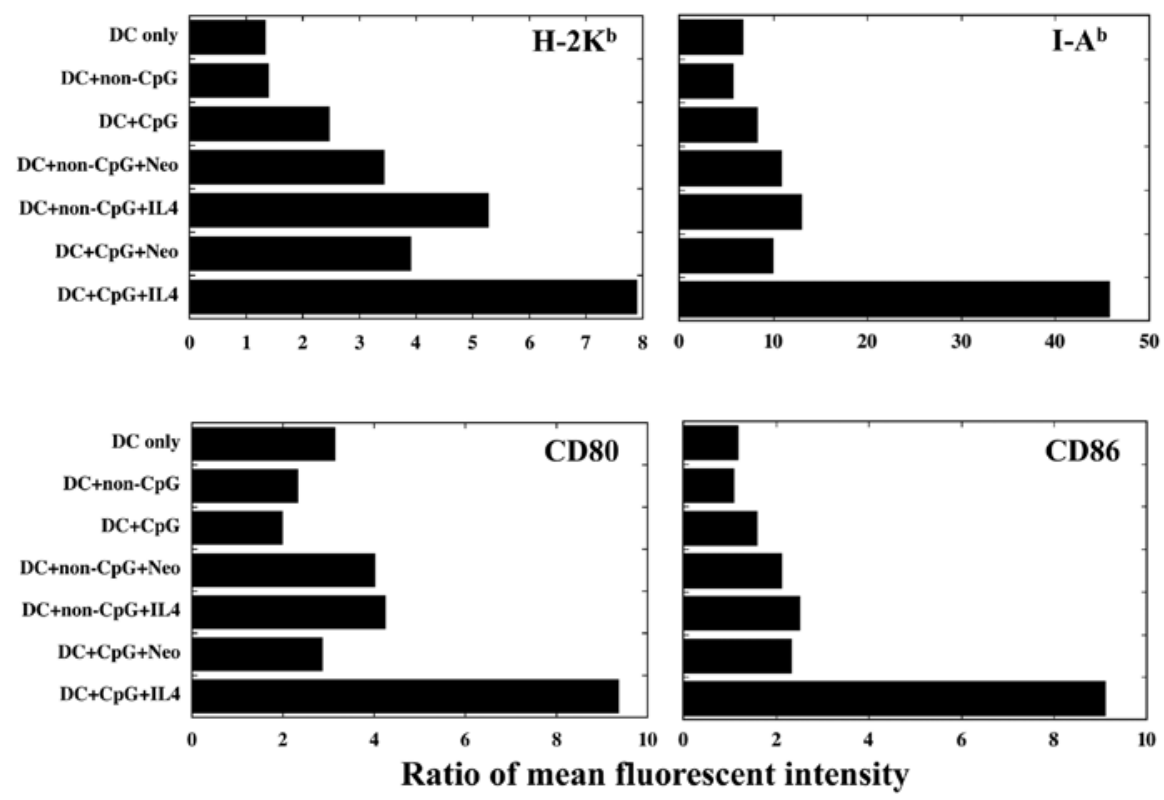

Figure 1. Coincubation with interleukin (IL)-4 and CpG-oligodeoxynucleotides (ODN) induces DC maturation in vitro. We incubated bone marrow-derived DCs with irradiated IL-4-overexpressing or control gene (Neo)-transduced MC38 cells either alone or in combination with CpG-ODN-1826 or non-CpGODN-1911 in vitro. Seven groups were compared: i) DC only, ii) DC + non-CpG, iii) DC + CpG, iv) DC + non-CpG + MC38-Neo, v) DC + non-CpG + MC38-IL4, vi) DC + CpG + MC38-Neo, and vii) DC + CpG + MC38-IL4. After a 24-h incubation, cells were stained with fluorescein isothiocyanate (FITC)conjugated anti-H-2 $\mathrm{K}^{\mathrm{b}}, \mathrm{I}-\mathrm{A}^{\mathrm{b}}, \mathrm{CD} 80$, and CD86 monoclonal antibodies. The results are shown as ratios of mean fluorescent intensity (MFI) of the incubated DCs to the MFI of FITC-conjugated control IgG of each group.

manner. Tumor size was measured twice a week using vernier calipers. Each experiment involved 4-6 mice per group. Mice with ulcerated tumors or tumors $>20 \mathrm{~mm}$ in diameter were sacrificed. Experiments with the therapeutic model were performed twice.

Immunohistological analysis. For leukocyte detection in tumor tissues, B6 mice were injected s.c. with $3 \times 10^{5} \mathrm{MC} 38-\mathrm{Neo}$ or -IL4 cells with or without $30 \mu \mathrm{g} /$ mouse of CpG-ODN-1826 in the area surrounding the established wild-type tumor 7 days after inoculation with $3 \times 10^{5}$ MC38-WT cells. Tumor tissues, which were harvested 3 days after inoculation with the genetically modified tumor. Serial $5-\mu \mathrm{m}$ sections were exposed to anti-Gr-1, anti-CD11c, anti-CD4, and anti-CD8a antibodies (Nippon Becton-Dickinson). Rat IgG2a was used as a control antibody. Immunostaining was completed with a Vectastain ABC kit (Vector Laboratories, Inc., Burlingame, CA). Immunoreactive cells were counted in 6 fields in a light microscope (magnification, $\mathrm{x} 400$ ) in a blinded fashion.

Induction of tumor-specific CTL. We assessed the tumorspecific cytolytic activity of immune mice. Mice were initially immunized with MC38-IL4 cells in combination with $30 \mu \mathrm{g}$ of either CpG-ODN-1826 or non-CpG-ODN-1911 on Day 0, and they were then inoculated with $3 \times 10^{5}$ MC $38-W T$ cells on Day 7. Subsequently, MC38-immune mice received a challenge of $1 \times 10^{6}$ MC38-WT cells on Day 28. Splenocytes $\left(3 \times 10^{6}\right.$ cells $\left./ \mathrm{ml}\right)$ were harvested from these mice on Day 42 , and they were then stimulated in vitro with irradiated (100 Gy) MC38-IL4 tumor cells ( $3 \times 10^{5}$ cells $\left./ \mathrm{ml}\right)$ and $6 \mu \mathrm{g} / \mathrm{ml}$ of nonCpG-ODN-1911 (IL-4 group) or IL4 cells in combination with $6 \mu \mathrm{g} / \mathrm{ml}$ of CpG-ODN-1826 (IL-4 + CpG group). Seven days later, responder cells $\left(1 \times 10^{6} \mathrm{cells} / \mathrm{ml}\right)$ were restimulated with
irradiated-IL4 tumor cells either alone (IL-4 group) or in combination with $6 \mu \mathrm{g} / \mathrm{ml}$ of CpG-ODN-1826 (IL-4 + CpG group) that was supplemented with irradiated syngeneic naive splenocytes (30 Gy, 1x10 cells $/ \mathrm{ml}$ ) as well as $50 \mathrm{IU} / \mathrm{ml}$ recombinant mouse IL-2 (Nippon Becton-Dickinson). Cytolytic assays were performed 6 days after the last stimulation using the responder cells as effector cells. Naïve splenocytes that were stimulated twice in vitro with irradiated-IL4 tumor cells and CpG-ODN as described above were used as control effector cells.

Cytolytic assays. Cytolytic assays were performed as previously described (22). Tumor-stimulated effector cells were assessed for cytolytic activity against MC38-WT and YAC-1 cells, which are sensitive to non-specific killing, in triplicate in 4-h ${ }^{51} \mathrm{Cr}$-release assays. The percentage of lysis was determined using the following formula: (release in assay - spontaneous release) x 100/(maximum release - spontaneous release). Maximum release was determined by the lysis of labeled target cells with $1 \%$ Triton X-100. Spontaneous release, which was measured by incubating target cells in the absence of effector cells, was $<10 \%$ of maximum release.

Statistical analyses. Significance was assessed by Student's t-tests. Differences between groups were considered significant when the $\mathrm{P}$-value was $<0.05$.

\section{Results}

Maturation of DCs was induced by coincubation with IL-4overexpressing tumor cells and $C p G-O D N$. We investigated the effects of IL-4 and CpG-ODN on DC maturation by flow cytometry. While the coincubation of DCs with MC38-IL4 


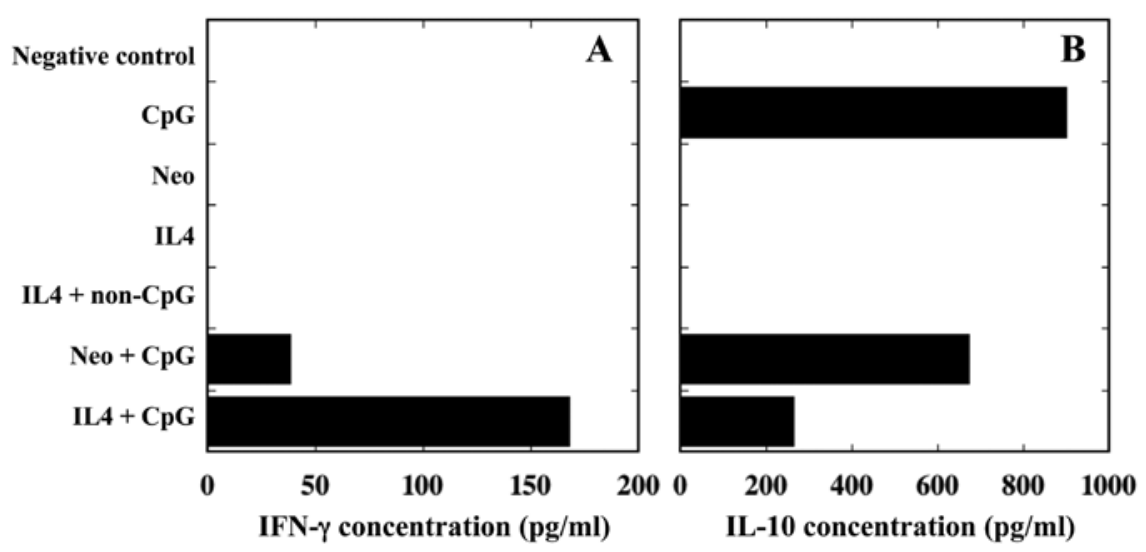

Figure 2. Type-1 T-helper (Th1) cytokine production by naïve splenocytes is enhanced by coincubation with IL-4 and CpG-ODN. Splenocytes from naive mice were stimulated with IL-4-overexpressing or control gene-transduced MC38 cells either alone or in combination with CpG-ODN-1826 or ODN-1911 in vitro. After a 24 -h incubation, we measured interferon (IFN)- $\gamma$ and IL-10 concentrations in the culture supernatant by enzyme-linked immunosorbent assay (ELISA).
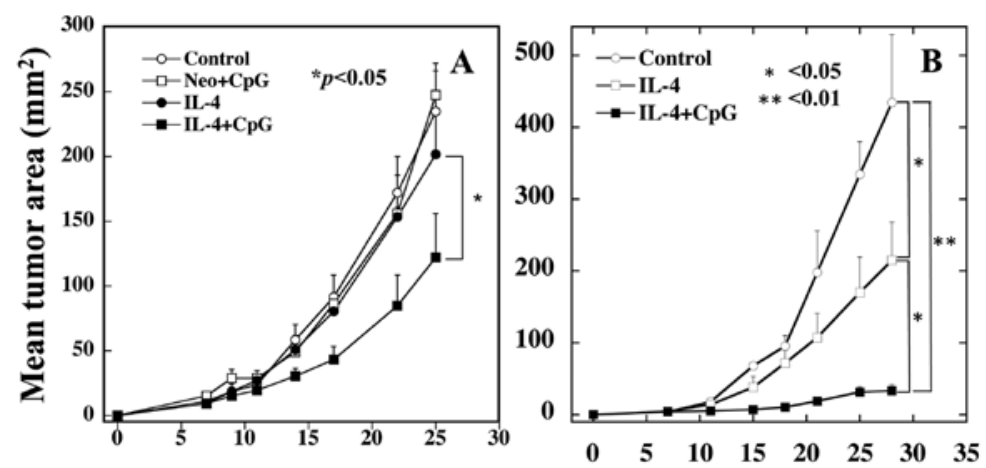

Days after tumor inoculation

Figure 3. The therapeutic inoculation of IL-4 and CpG-ODN suppresses the growth of parental tumors in vivo. B6 mice were injected with MC38-WT cells in the right flank. (A) Seven, 9 and 11 days after the WT inoculation, 3x10 $10^{5}$ cells/mouse MC 38-Neo (Control; open circles), MC 38-IL4 cells with 30 $\mu \mathrm{g} / \mathrm{mouse}$ CpG-ODN-1826 (Neo + CpG; open squares), MC38-IL4 cells with ODN-1911 (IL-4; closed circle), or MC38-IL-4 cells with CpG-ODN-1826 (IL-4 + CpG; closed squares) were inoculated around the established parental tumors, which had reached $4-20 \mathrm{~mm}^{2}$ in size. (B) Otherwise, tumor-bearing B6 mice were inoculated 5x10 cells/mouse MC38-Neo (Control; open circles) or MC38-IL4 cells with either ODN-1911 (IL-4; open squares) or CpG-ODN-1826 (IL-4 + $\mathrm{CpG}$; closed squares) were inoculated in the same manner. Tumor size was measured twice a week. Results are reported as mean tumor area $\left(\mathrm{mm}^{2}\right) \pm \mathrm{SE}$. Significance at $95 \%$ confidence limits is indicated.

enhanced the expression of MHC class I, class II, and costimulatory molecules, only minimal phenotypic changes in DCs were detected after coincubation with CpG-ODN. When we compared DCs that were incubated with IL-4-overexpressing MC38 cells and CpG-ODN-1826 (DC + CpG + IL-4) with the other groups, the expression of $\mathrm{H}-2 \mathrm{~K}^{\mathrm{b}}, \mathrm{I}-\mathrm{A}^{\mathrm{b}}, \mathrm{CD} 80$, and CD86 molecules on the DCs was clearly upregulated (Fig. 1). These results suggest that IL-4 and CpG-ODN have synergistic effects on DC maturation.

The combination of IL-4-overexpressing MC38 cells and CpG-ODN promotes the production of Thl-type cytokines by naïve splenocytes in vitro. Since the combination of IL-4 and CpG-ODN enhanced DC maturation, we observed their effects on naïve splenocytes. ELISAs confirmed that naïve splenocytes produced high amounts of IFN- $\gamma$ after a $24-\mathrm{h}$ coincubation with MC38-IL4 and CpG-ODN-1826 (Fig. 2A), whereas IFN- $\gamma$ production could not be detected after coincubation with either IL-4 or CpG-ODN alone. While IL-10 production was clearly detected when naïve splenocytes were incubated with CpG-ODN (Fig. 2B), coincubation with MC38-IL4 suppressed the production of IL-10. These results suggest that the combination of IL-4 and CpG-ODN promoted Th1-type immune responses.

Therapeutic inoculation of IL-4-overexpressing MC38 cells and $C p G-O D N$ reduces the outgrowth of established tumors in vivo. Next, we observed whether CpG-ODN had additive antitumor effects on the effects of IL- 4 on established tumors in vivo. We treated tumor-bearing mice with MC38-IL4 and CpG-ODN-1826 and compared the parental tumor size. As shown in Fig. 3A, the combination therapy of MC38-IL4 ( $3 \times 10^{5}$ cells/mouse) and CpG-ODN (30 $\mu \mathrm{g} /$ mouse) significantly reduced the outgrowth of the parental tumors (IL-4 vs. IL-4 + $\mathrm{CpG}, \mathrm{P}=0.048$ ) whereas treatment with MC38-Neo + CpG and MC38-IL4 + non-CpG did not show any antitumor effects. Furthermore, the combination

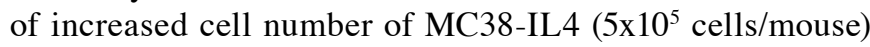



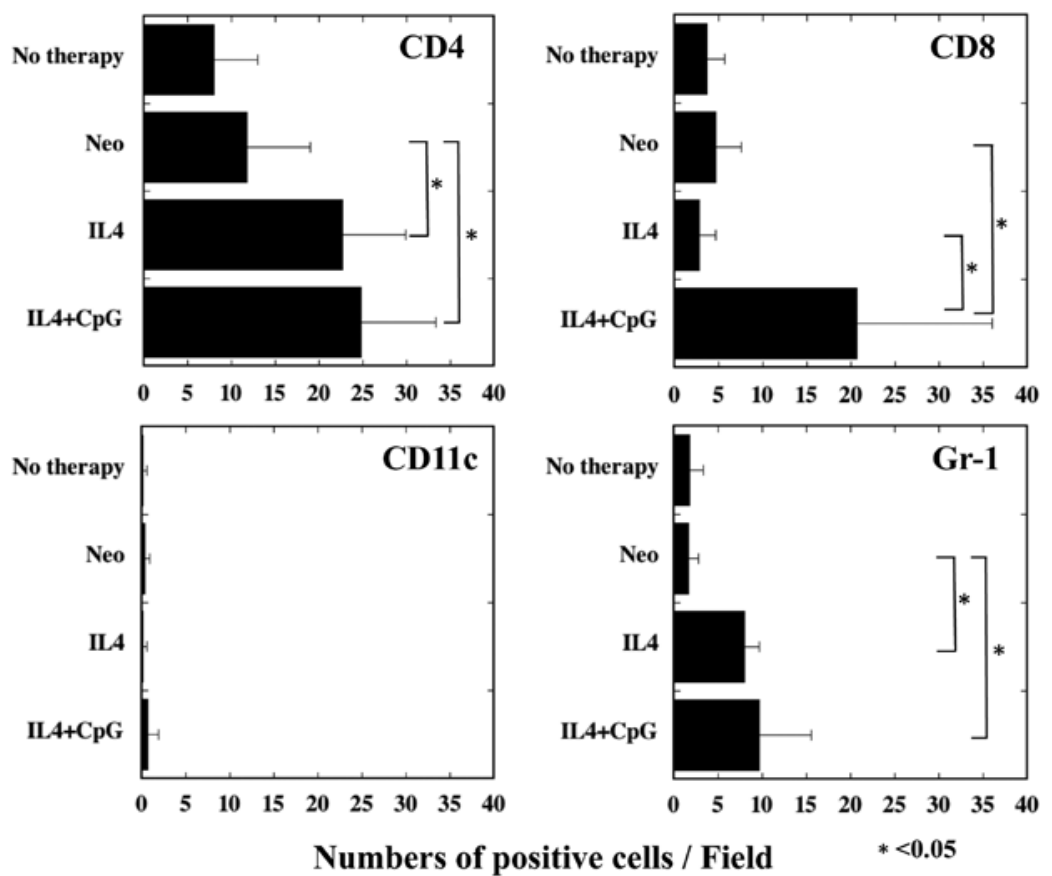

Figure 4. $\mathrm{CD} 88^{+}$cells significantly infiltrate the established wild-type (WT) tumors of mice treated with MC38-IL4 and CpG-ODN. B6 mice were injected with either MC38-Neo or MC38-IL4 cells with or without CpG-ODN-1826 in the area surrounding the established WT tumor 7 days after inoculation of MC38-WT cells. Tumor tissues were harvested 3 days after inoculation of the genetically modified tumor and immediately embedded in optimal cutting temperature compound and frozen for sectioning. Serial $5-\mu \mathrm{m}$ sections were exposed to anti-Gr-1, anti-CD11c, anti-CD4, or anti-CD8a antibodies. Rat IgG2a was used as a control antibody. Immunostaining was completed with a Vectastain ABC kit. Immunoreactive cells were counted in 6 fields in a light microscope (magnification, $\mathrm{x} 400$ ) in a blinded fashion. Results are shown as mean numbers of positive cells/field $\pm \mathrm{SD}$.

and CpG-ODN (30 $\mu \mathrm{g} / \mathrm{mouse})$ was more effective against the established tumors (Fig. 3B; IL-4 vs. IL-4 + CpG, P=0.015) while treatment with MC38-IL4 (5x $10^{5}$ cells/mouse) only also revealed antitumor activity. Therefore, the combination therapy of IL-4 and CpG-ODN has an additive antitumor effect in vivo.

Treatment of IL-4-overexpressing MC38 cells with the combination of CpG-ODN induces the infiltration of CD8-positive cells in established wild-type tumors. We analyzed the mechanisms of the antitumor effects that were induced by the inoculation of MC38-IL4 cells with CpG-ODN-1826. As shown in Fig. 4, WT tumors of mice treated with MC38-IL4 showed significant infiltration with $\mathrm{Gr}-1^{+}$cells, as previously reported (4). More $\mathrm{CD}^{+}$cells infiltrated tumors in mice treated with MC38-IL4 alone, as well as with MC38-IL4 and $\mathrm{CpG}-\mathrm{ODN}$, compared with those in mice treated with MC38-Neo ( $\mathrm{P}=0.039$ and $\mathrm{P}=0.026$, respectively). While only a few $\mathrm{CD}^{+}$cells could be detected in the tumors of mice treated with MC38-IL4 alone, a marked infiltration of $\mathrm{CD}^{+}$cells was observed in the tumors of mice treated with MC38-IL4 and CpG-ODN (Fig. 4; MC38-IL4 alone: $2.8 \pm 1.9$ cells/field vs. MC38-IL4 + CpG-ODN, 20.7 \pm 15.3 cells/field, $\mathrm{P}=0.027$ ). $\mathrm{CD} 11 \mathrm{c}^{+}$cells were rarely seen in any of the groups. These results suggest that the initial antitumor effects induced by MC38-IL4 and CpG-ODN may be dependent on CD8 ${ }^{+}$and $\mathrm{CD} 4^{+}$cells.

Potent tumor-specific cytolysis is detected when splenocytes of MC38-immune mice are stimulated by the combination of

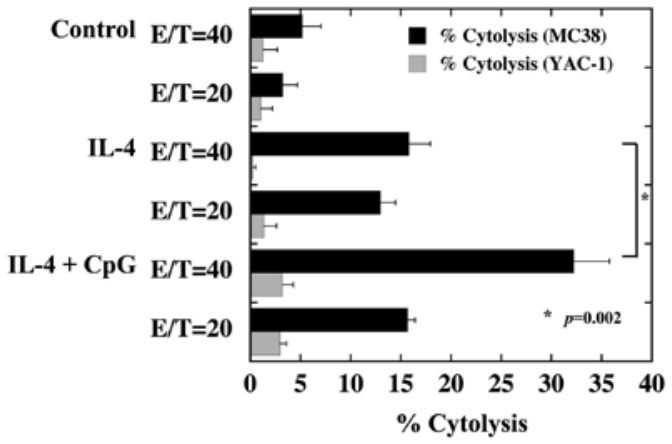

Figure 5. Stimulation with MC38-IL4 and CpG-ODN induces potent tumorspecific cytolysis. Immune mice received injections of both MC38-IL4 cells and control-ODN-1911 (IL-4) or MC38-IL4 cells in combination with CpG-ODN-1826 (IL4 + CpG) on Day 0 and MC38-WT cells on Days 7 and 28. Splenocytes from immunized mice were harvested on Day 42 and stimulated in vitro with irradiated MC38-IL4 tumor cells either alone (IL-4) or in combination with CpG-ODN-1826 (IL4 + CpG). Seven days later, responder cells were restimulated with irradiated-IL4 tumor cells either alone (IL-4) or in combination with CpG-ODN-1826 (IL4 + CpG) and supplemented with irradiated syngeneic naive splenocytes as well as recombinant mouse IL-2. Naïve splenocytes that were stimulated with irradiated-IL4 tumor cells and $\mathrm{CpG}-\mathrm{ODN}$ as described above were also used as control effector cells (Control). A cytolytic assay $\left(4 \mathrm{~h}-{ }^{51} \mathrm{Cr}\right.$-release assay) against MC38 or YAC-1 cells was performed 6 days after the second stimulation. Results are reported as mean $\%$ cytotoxicity \pm SD. E:T, effector to target.

$I L$-4-overexpressing tumor cells and $C p G$-ODN. Because the combination of IL-4 and CpG-ODN seemed to induce potent Th1-type immune responses, we tried to detect the generation of tumor-specific CTL in mice immunized with IL-4 and CpG-ODN. As shown in Fig. 5, effector cells that were stimu- 
lated with IL-4 and CpG-ODN revealed marked cytolysis that was specific for MC38-WT cells $(32.2 \pm 3.5 \%$ for MC38 vs. $3.2 \pm 1.1 \%$ for YAC-1; at effector to target ratio, $\mathrm{E}: \mathrm{T}=40$ ). Although tumor-specific cytolysis was also detected when the splenocytes of immunized mice that had been inoculated with MC38-IL4 cells and non-CpG-ODN-1911 in vivo were stimulated with MC38-IL4 cells in vitro, the specific lysis was lower compared to that of the splenocytes of mice treated with IL-4 and $\mathrm{CpG}-\mathrm{ODN}(15.8 \pm 2.1 \%$ for MC38 vs. $0.3 \pm 0.3 \%$ for YAC-1; $\mathrm{E}: \mathrm{T}=40, \mathrm{P}=0.002$ when compared with IL4 $+\mathrm{CpG}$ for lysis of MC38). Naïve splenocytes stimulated with IL-4 + CpG-ODN did not show any MC38-specific killing (Fig. 5). The results suggested that immunization with IL-4 and CpG-ODN effectively induce tumor-specific immune response in vivo.

\section{Discussion}

In this study, we observed that the combination of IL-4 and CpG-ODN enhanced the expression of MHC and costimulatory molecules on the surface of DCs. In addition, the combination promoted IFN- $\gamma$ production and suppressed IL-10 production in naïve splenocytes in vitro, which strongly suggested that the combination of IL-4 and CpG-ODN contributed to T-cell differentiation towards a Th1-type immune response. The combined therapy of IL-4 and CpG effectively suppressed the outgrowth of parental tumors in vivo compared to IL-4 monotherapy. The results of both the immunohistochemical and the tumor-specific cytolysis analyses suggested that Th1-type immune responses were strongly induced in mice treated with IL-4 and CpG-ODN.

IL-4, which is a representative Th2-type cytokine, is produced by Th2-type cells. The Th2-type response is thought to suppress the generation of Th1 cells (23), which are usually involved in antitumor immunity. However, it has been reported that IL-4 shows multiple suppressive effects on tumors, and our previous investigation showed that IL-4 gene transduction in MC38 cells did not affect in vitro tumor growth, while tumors were not seen in most mice injected with IL-4-overexpressing MC38 cells. This observation implies that IL-4 does not injure MC38 cells directly but reduces their tumorigenicity by inducing host immune responses. Our previous study and other investigations have suggested that IL-4 recruits and activates granulocytes in the microenvironment of parental tumors in order to attack and kill the tumor cells during the primary response. However, how IL-4 recruits or stimulates granulocytes in the tumor microenvironment remains unclear $(4,24)$. Thus, IL-4 induces tumor-specific cellular immune responses, which contribute to long-lasting immunity against the parental tumors.

For further improvement of the antitumor effects of IL-4based immune therapy, we performed combination therapy, which consisted of IL-4 with CpG-ODN, and this combination has been reported as a potent inducer of immune responses. As previously reported, treatment with $\mathrm{CpG-ODN}$ alone did not reduce tumor outgrowth (3). Although CpG-ODN is thought to promote the induction of Th1-type cytokines, CpG-ODN promoted IL-10 production by naïve splenocytes in our system. Reports show that CpG induces Th2-type cytokines in certain conditions support our observation $(25,26)$. MC38-IL4 did not promote IL-10 production, whereas exogenous IL-4 might. We speculate that irradiated tumor cells modified the immune circumstances in the culture. In any case, we suggest that the balance of $\mathrm{Th} 1 / \mathrm{Th} 2$ cytokine production is critical to the induction of potent immune responses in the early stages of tumor development and that the great suppression of outgrowth of the parental tumors that was seen in this study occurred because the combination of IL-4 and CpG enhanced IFN- $\gamma$ production and suppressed IL-10 production. Further investigation is required in order to clarify the mechanisms by which IL-4 reduces the IL-10 production that is induced by $\mathrm{CpG}$.

However, opposite effects of IL-4 on tumors have also been reported. IL-4 itself may affect tumor growth, especially on those that have abundant IL-4 receptors. The levels of expression of the IL-4 receptor correlated with the tumorigenic potential in a murine model (27). Several tumors appeared to be resistant to apoptosis, which is induced by chemotherapeutic agents or CD95 ligation, in an IL-4-dependent manner. IL-4 seemed to modify the immunological functions of effector cells. The cultivation of naïve $\mathrm{CD}^{+} \mathrm{T}$ cells in the presence of IL-4 resulted in poor cytolytic function in the cells by reducing the levels of perforin and granzymes $(27,28)$. Whether an immune response is predominantly Th1 or Th2 may depend on a number of other factors, such as mouse strain, kind of tumor, amount of tumor antigen, and the amount of cytokine $(29,30)$.

It has been reported that the combination of $\mathrm{CpG}, \mathrm{IL}-4$, and CD40 ligand effectively induce CTLs by enhancing the expression of immunogenic molecules on B-cell precursor acute lymphoblastic leukemia cells (31). Although MHC class I molecules were slightly upregulated by IL-4 transduction in MC38 cells, changes in other molecules, such as costimulatory and MHC class II molecules, could not be detected in our system (unpublished data). It was thought that IL-4 and CpG-ODN resulted in antitumor effects by inducing Th1-type immune responses, especially that of tumor-specific CTLs, rather than by modulating the immunogenic molecules in the tumor cells. The mechanism by which cytokines and immunomodulators exhibit antitumor effects may also depend on the models used in the experiments.

In order to perform tumor-based gene therapy, there are a number of issues, such as dose determination, site of therapeutic injection, and therapeutic interval, that need to be determined, but the most critical issue is the establishment of the patient's cytokine gene-transduced tumor cells. The development of a new technique that transduces a targeted gene to a patient's cells may be required.

Finally, we found in this study that combination therapy with IL-4 and CpG-ODN had potent antitumor effects on established tumors through the induction of potent Th1-type immune responses in the hosts. Thus, this combination therapy may be a candidate for clinical cancer therapy, but further investigation is needed before clinical trials.

\section{Acknowledgements}

This study was supported in part by a grant from the Ministry of Health, Labor, and Welfare of Japan (K. Hiroishi); a Grant-in-Aid for Scientific Research (C) from the Ministry of Education, Culture, Sports, Science and Technology of 
Japan (K. Hiroishi); a grant for the High-Technology Research Center Project from the Ministry of Education, Culture, Sports, Science, and Technology of Japan (M. Imawari); and the Research Program of Intractable Disease provided by the Ministry of Health, Labor and Welfare of Japan (K. Hiroishi).

\section{References}

1. Eguchi J, Hiroishi K, Ishii S and Mitamura K: Interferon-alpha and interleukin-12 gene therapy of cancer: interferon-alpha induces tumor-specific immune responses while interleukin-12 stimulates non-specific killing. Cancer Immunol Immunother 52: 378-386, 2003

2. Ishii S, Hiroishi K, Eguchi J, Hiraide A and Imawari M: Dendritic cell therapy with interferon-alpha synergistically suppresses outgrowth of established tumors in a murine colorectal cancer model. Gene Ther 13: 78-87, 2006.

3. Hiraide A, Hiroishi K, Eguchi J, Ishii S, Doi H and Imawari M: Dendritic cells stimulated with cytidine-phosphate-guanosine oligodeoxynucleotides and interferon-alpha-expressing tumor cells effectively reduce outgrowth of established tumors in vivo. Cancer Sci 99: 1663-1669, 2008.

4. Eguchi J, Hiroishi K, Ishii S, et al: Interleukin-4 gene transduced tumor cells promote a potent tumor-specific Th1-type response in cooperation with interferon-alpha transduction. Gene Ther 12 733-741, 2005

5. Seder RA, Paul WE, Davis MM and Fazekas de St Groth B: The presence of interleukin 4 during in vitro priming determines the lymphokine-producing potential of $\mathrm{CD}^{+} \mathrm{T}$ cells from $\mathrm{T}$ cell receptor transgenic mice. J Exp Med 176: 1091-1098, 1992.

6. Hsieh CS, Heimberger AB, Gold JS, O'Garra A and Murphy KM: Differential regulation of $\mathrm{T}$ helper phenotype development by interleukins 4 and 10 in an alpha beta T-cell-receptor transgenic system. Proc Natl Acad Sci USA 89: 6065-6069, 1992.

7. Paul WE: Interleukin 4/B cell stimulatory factor 1: one lymphokine, many functions. FASEB J 1: 456-461, 1987.

8. Li Z, Chen L and Qin Z: Paradoxical roles of IL-4 in tumor immunity. Cell Mol Immunol 6: 415-422, 2009.

9. Toi M, Bicknell R and Harris AL: Inhibition of colon and breast carcinoma cell growth by interleukin-4. Cancer Res 52: 275-279, 1992.

10. Obiri NI, Hillman GG, Haas GP, Sud S and Puri RK: Expression of high affinity interleukin- 4 receptors on human renal cell carcinoma cells and inhibition of tumor cell growth in vitro by interleukin-4. J Clin Invest 91: 88-93, 1993.

11. Volpert OV, Fong T, Koch AE, et al: Inhibition of angiogenesis by interleukin 4. J Exp Med 188: 1039-1046, 1998.

12. Rosenman SJ, Shrikant P, Dubb L, Benveniste EN and Ransohoff RM: Cytokine-induced expression of vascular cell adhesion molecule-1 (VCAM-1) by astrocytes and astrocytoma cell lines. J Immunol 154: 1888-1899, 1995.

13. Yu JS, Wei MX, Chiocca EA, Martuza RL and Tepper RI: Treatment of glioma by engineered interleukin 4-secreting cells. Cancer Res 53: 3125-3128, 1993.

14. Benedetti S, Dimeco F, Pollo B, et al: Limited efficacy of the HSV-TK/GCV system for gene therapy of malignant gliomas and perspectives for the combined transduction of the interleukin-4 gene. Hum Gene Ther 8: 1345-1353, 1997.
15. Saleh M, Wiegmans A, Malone Q, Stylli SS and Kaye AH: Effect of in situ retroviral interleukin-4 transfer on established intracranial tumors. J Natl Cancer Inst 91: 438-445, 1999.

16. Eguchi J, Kuwashima N, Hatano M, et al: IL-4-transfected tumor cell vaccines activate tumor-infiltrating dendritic cells and promote type-1 immunity. J Immunol 174: 7194-7201, 2005.

17. Okada H, Lieberman FS, Walter KA, et al: Autologous glioma cell vaccine admixed with interleukin-4 gene transfected fibroblasts in the treatment of patients with malignant gliomas. J Transl Med 5: 67, 2007.

18. Kurtz DM, Tschetter LK, Allred JB, et al: Subcutaneous interleukin-4 (IL-4) for relapsed and resistant non-Hodgkin lymphoma: a phase II trial in the North Central Cancer Treatment Group, NCCTG 91-78-51. Leuk Lymphoma 48: 1290-1298, 2007.

19. Hiraide A, Hiroishi K, Ishii S, Eguchi J and Imawari M: Cytidine-phosphate-guanosine oligodeoxynucleotides and interferon-alpha-expressing tumor cells effectively induce dendritic cell maturation in vitro. Anticancer Res 26: 211-218, 2006.

20. Pear WS, Nolan GP, Scott ML and Baltimore D: Production of high-titer helper-free retroviruses by transient transfection. Proc Natl Acad Sci USA 90: 8392-8396, 1993.

21. Hiroishi K, Tuting T, Tahara H and Lotze MT: Interferon-alpha gene therapy in combination with CD80 transduction reduces tumorigenicity and growth of established tumor in poorly immunogenic tumor models. Gene Ther 6: 1988-1994, 1999.

22. Hiroishi K, Tuting T and Lotze MT: IFN-alpha-expressing tumor cells enhance generation and promote survival of tumor-specific CTLs. J Immunol 164: 567-572, 2000.

23. Sher A and Coffman RL: Regulation of immunity to parasites by T cells and T cell-derived cytokines. Annu Rev Immunol 10: 385-409, 1992

24. Noffz G, Qin Z, Kopf M and Blankenstein T: Neutrophils but not eosinophils are involved in growth suppression of IL-4-secreting tumors. J Immunol 160: 345-350, 1998.

25. Triozzi PL, Aldrich W and Ponnazhagan S: Regulation of the activity of an adeno-associated virus vector cancer vaccine administered with synthetic Toll-like receptor agonists. Vaccine 28: 7837-7843, 2010

26. Fitzner N,Zahner L, Habich C and Kolb-Bachofen V: Stimulatory type A CpG-DNA induces a Th2-like response in human endothelial cells. Int Immunopharmacol 11: 1789-1795, 2011.

27. Li Z, Jiang J, Wang Z, et al: Endogenous interleukin-4 promotes tumor development by increasing tumor cell resistance to apoptosis. Cancer Res 68: 8687-8694, 2008.

28. Kienzle N, Olver S, Buttigieg K, et al: Progressive differentiation and commitment of $\mathrm{CD} 8^{+} \mathrm{T}$ cells to a poorly cytolytic CD8low phenotype in the presence of IL-4. J Immunol 174: 2021-2029, 2005.

29. Allen JE and Maizels RM: Th1-Th2: reliable paradigm or dangerous dogma? Immunol Today 18: 387-392, 1997.

30. Schuler T, Qin Z, Ibe S, Noben-Trauth N and Blankenstein T: $\mathrm{T}$ helper cell type 1-associated and cytotoxic T lymphocytemediated tumor immunity is impaired in interleukin 4-deficient mice. J Exp Med 189: 803-810, 1999.

31. Fabricius D, Breckerbohm L, Vollmer A, et al: Acute lymphoblastic leukemia cells treated with $\mathrm{CpG}$ oligodeoxynucleotides, IL-4 and CD40 ligand facilitate enhanced anti-leukemic CTL responses. Leukemia 25: 1111-1121, 2011. 